\title{
Steady-State Impedance Mapping in Grids with Power Electronics: What Is Grid Strength in Modern Power Systems?
}

\author{
Oriol Gomis-Bellmunt ${ }^{a, *}$, Jie Song ${ }^{a}$, Marc Cheah-Mane ${ }^{a}$ and Eduardo Prieto-Araujo ${ }^{a}$ \\ ${ }^{a}$ Departament d'Enginyeria Elèctrica, Centre d'Innovació Tecnològica en Convertidors Estàtics i Accionaments (CITCEA-UPC), Universitat Politècnica \\ de Catalunya, Barcelona 08028, Spain
}

\section{ARTICLE INFO}

\section{Keywords:}

Grid equivalent

Grid strength

Impedance mapping

Power electronics dominated power systems

\begin{abstract}
A B S T R ACT
The paper analyzes grid strength and grid impedance for systems with significant penetration of power electronics. The work shows that the traditionally employed Thévenin equivalent cannot capture the different saturation states of power converters and therefore some classical definitions of grid strength based on short-circuit power at the point of connection fail to describe the system behavior. The paper proposes to map the impedance for all the different possible voltages at the point of connection and analyzes how the impedance depend on the voltage and angle of derivation for different possible simplified systems (grid-following converter in parallel with a Thévenin equivalent and gridforming converter) considering the different saturation states of the converter. Numerical results and case studies are included as examples of the conducted analysis.
\end{abstract}

\section{Introduction}

Modern power systems are increasingly populated with power electronics converters. Power converters are present in the prevailing renewable energy generators (wind and solar PV), energy storage interfaces, HVDC transmission systems, Flexible AC Transmission Systems (FACTS), electrical vehicle chargers and industrial drives. Power converters are different from synchronous generators as they are fully controllable devices with very limited overload capability. This implies important challenges related to power system operation (related to the very low inertia) and protection (limited short-circuit current). Steady-state power system calculations and short-circuit analyses are typically conducted [1,2] assuming there is a slack bus in the network which can provide the needed current to ensure equilibrium. In power systems dominated by power electronics, this assumption is not true anymore. One possible approach suggested by some recent research projects is to conduct dynamic simulations to understand system behaviour in different conditions, including fault scenarios. Several electromagnetic transient (EMT) test cases have been presented in [3] to study the power systems with high penetration of power electronics. The resonance between HVDC link and $\mathrm{AC}$ networks has been analyzed in [4]. The operation of several HVDC links in real applications has been studied in both normal operation and fault conditions using real-time simulations [5]. However, a steady-state analysis presents a more efficient approach in some studies.

The assessment of grid equivalent impedance and grid strength is a fundamental but essential task for the analysis and design of power systems. The grid code usually sets the lower limit to the grid strength to ensure the stability of

This work was supported in part by FEDER / Ministerio de Ciencia, Innovación y Universidades - Agencia Estatal de Investigación, Project RTI2018-095429-B-I00 and in part by FI-AGAUR Research Fellowship Program, Generalitat de Catalunya.

*Corresponding author

@oriol.gomis@upc.edu (Oriol Gomis-Bellmunt) both AC and DC transmission networks [6-9]. This is especially critical for the system with significant integration of renewable generations as they are considered as sensitive to voltage variations $[10,11]$. Also, an accurate estimation of grid impedance enables a proper design of power converter control $[12,13]$.

Grid strength analysis in modern power systems has also been reported in the literature. The conventional ShortCircuit Ratio (SCR) has been modified into a weighted format to include the contribution from different generating units in order to give a more precise estimation of grid strength of large-scale wind power plants [14]. A multiinfeed SCR has been proposed as a grid strength index of hybrid AC/DC transmission systems [15, 16]. The effective SCR is applied to analyze power systems dominated by power converters in [17-19]. A generalized SCR has been proposed to assess the grid strength of power electronics populated power systems [20]. This generalized SCR has been applied to analyze the stability of multi-infeed line commutated converter (LCC) based HVDC systems [21] and wind farms [22]. The methodologies presented in [14-22] are more suitable for analysis of modern power systems compared to conventional SCR as they have included the impact of power converters control to the grid strength. However, the potential current-saturated operation of power converters are not considered in these references and therefore they might be limited for some studies. This is especially critical for short-circuit analysis as several or all converters might be operated with a saturated current.

A completely different approach looking at this problem from a different perspective is taken in the present work, considering the possible saturation of different converters in the network. Furthermore, as it can be seen that the grid cannot be synthesized by the so-called Thévenin equivalent (commonly used in power system studies), an alternative concept based on voltage to current mapping is adopted. This new equivalent allows conducting steady-state and short-circuit studies of modern power systems considering 
the saturation state of different converters.

The present paper delves into the analysis of the grid impedance as it is a key quantity for understanding the strength of the grid related to the grid capacity of maintaining the desired voltage whenever the current changes. The work explores the equivalent grid impedance considering voltage-current characteristics which analyzes the circuit overcoming the limitations and deviations obtained when using the Thévenin equivalent. Impedance maps are obtained showing how the impedance changes for different operating points. This work suggests to use such impedance map to characterize the strength of the grid in order to provide reliable information when characterizing power systems dominated by power electronics.

The rest of the paper is organized as follows. Section 2 presents equivalent system for traditional and modern power systems. Section 3 defines and elaborates the concepts of impedance mapping and grid strength in power systems with power electronics. A methodology to obtain the impedance map is presented in Section 4. The equations and methodologies presented are applied in selected case studies in Section 5. The conclusions are summarized in Section 6. All the equations of the paper as well as the implemented calculations are expressed in the per-unit system.

\section{Equivalents for modern power systems}

Grid strength is a concept that can be used to understand power flows and interactions in power systems. In particular, the grid strength from a specific bus can be characterized in terms of the grid impedance or the system inertia [23]. When grid impedance is considered, grid strength is related to the capacity of a system to maintain the voltage against external perturbations. This basically means that a strong (stiff) grid is capable of keeping the voltage at the desired value when the current changes. A weak grid will experience large voltage deviations when the current changes and therefore it can become unstable or can bring the voltage to non-acceptable values in some conditions. When the system inertia is considered, grid strength is related to the capacity of a system to maintain the frequency against external perturbations. This work focuses on voltage grid strength, related to impedance.

In traditional power systems (see Fig. 1) dominated by synchronous generators, the overall grid can be synthesized in a grid equivalent which is commonly referred to as Thévenin equivalent. This equivalent is based on a voltage source (Thévenin voltage or open-circuit voltage) behind an impedance (Thévenin impedance), which characterizes the studied system as seen from the Point of Connection (POC).

For any external system connected to a Thévenin equivalent (as shown in Fig. 1 bottom) the voltage-current equation can be defined as $\underline{u}=\underline{u}_{t h}-\underline{z}_{t h} \underline{i}$.

Doing a small current increase $\Delta \underline{i}$, a voltage increase $\Delta \underline{u}$ can be obtained as $\underline{u}+\Delta \underline{u}^{\prime}=\underline{u}_{t h}-\underline{z}_{t h}(\underline{i}+\Delta \underline{i})$. Combin-

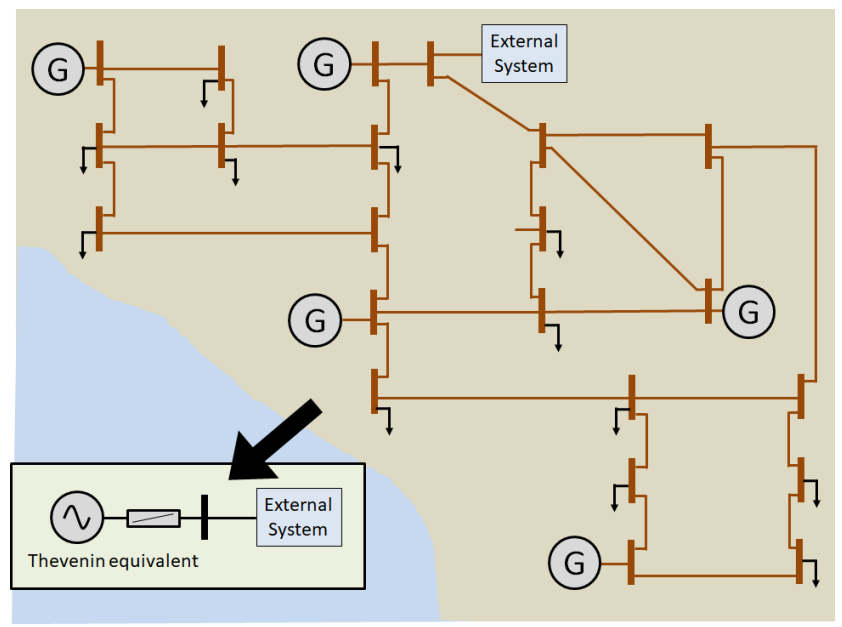

Figure 1: Traditional power system dominated by synchronous generators and grid equivalent based on a Thévenin equivalent.

ing the previous equations, the Thévenin equivalent can be identified from measurements around an equilibrium point, $(\underline{u}, \underline{i})$, as

$$
\underline{z}_{t h}=-\frac{\Delta \underline{u}}{\Delta \underline{i}} \text { and } \underline{u}_{t h}=\underline{u}-\frac{\Delta \underline{u}}{\Delta \underline{i}} i
$$

For a system with a given Thévenin equivalent, the previous equations will be satisfied for all the equilibrium points. For a short-circuit, $\underline{u}=0$ and $\underline{i}_{s c}=\underline{u}_{t h} / \underline{z}_{t h}$. In this case, short-circuit power is defined as the power injected by the Thévenin source as $\underline{s}_{s c c}=\underline{u}_{t h} \underline{i}_{s c}^{*}=u_{t h}^{2} / \underline{z}_{t h}^{*}$. As the short circuit power $\underline{s}_{s c c}$ is clearly related to the Thévenin impedance $\underline{z}_{t h}$ for a given Thévenin voltage (open-circuit voltage) $\underline{u}_{t h}$, power engineers commonly use short circuit power as the relevant quantity to refer to the strength of a network [24]. Furthermore, the short-circuit ratio (SCR) is defined for power systems in order to compare the grid strength of AC systems in relation to the impedance from a specific bus where a new element is going to be connected. SCR can be expressed as $[23,25] S C R=s_{\text {sc }} / p_{\text {elem }}$ where $s_{s c}$ is the short circuit power of the ac system and $p_{\text {elem }}$ is the power rating of the new element connected to the bus. When connecting a power converter to the power system, a typical approach is to calculate the SCR to have a clear understanding on how strong the grid is compared to new converter connected to it. Low SCR indicate weak grids and potential challenges in the integration of the converter.

In power system with significant penetration of power electronics (see Fig. 2), the Thévenin equivalent fails to describe the behaviour of the system, because of the different converter control modes and current saturation states. As an example, whenever there is a short-circuit, converters remain connected to the grid and inject reactive current up to nominal converter current following the requirements of the grid codes. In this case, converters behave as current sources while in normal conditions they were regulating the current to exchange the desired power between converter and grid. 
This paper characterizes power systems with penetration of power electronics considering different saturation states of power converters. As it shown in Fig. 2 (bottom), the grid equivalent can be described by a function mapping the voltage to the current (similar approaches could be derived inversely, mapping the current to the voltage) as $\underline{i}=F(\underline{u})$. In this case, the equivalent impedance of the system can still define the relationship between voltage and current (taking into account that this equivalent impedance is different in different operation points $(\underline{u}, \underline{i})$ ). In any case, the relationship between short-circuit power and impedance does not hold. Therefore, in order to express the strength of the network, an analysis on how the grid equivalent impedance changes for different operation points is needed.

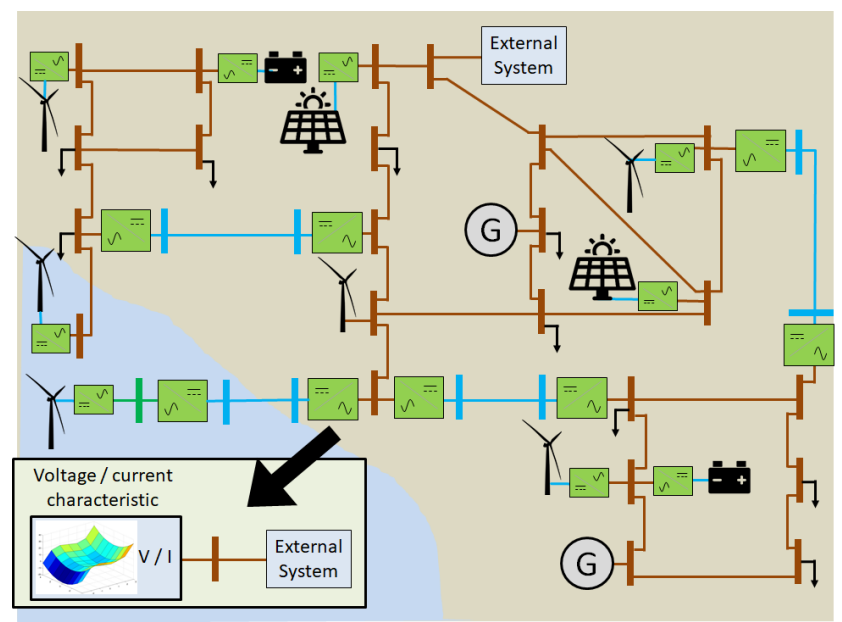

Figure 2: Modern power system with significant penetration of power electronics, and equivalent system based on voltage to current mapping.

Fig. 4 shows the voltage to current characteristic of an ideal Thévenin equivalent (Fig. 3), where each voltage $\underline{u}=$ $u_{x}+j u_{y}$ is mapped to a current $\underline{i}=i_{\angle \alpha-i}$. Fig. 6 shows an example of a voltage to current characteristic of a VSC in PQ control connected in parallel with AC grid Thévenin equivalent with the scheme shown in Fig. 5. The voltagecurrent characteristics present three different converter saturation states (Unsaturated (USS), Partially Saturated (PSS) and Fully Saturated (FSS)), that will be analyzed in Section 3). Fig. 8 shows the voltage to current characteristic of a grid-forming converter (Fig. 7), which is equivalent to the Thévenin equivalent until the converter saturates. When this happens, the current is limited to the nominal value. In this case, the $U-I$ characteristic shows two states: USS and FSS. Similar $U-I$ characteristics can be derived for more complex systems.

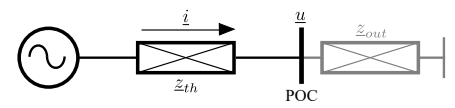

Figure 3: Scheme of a Thévenin equivalent

The grid strength can be defined as the grid capacity

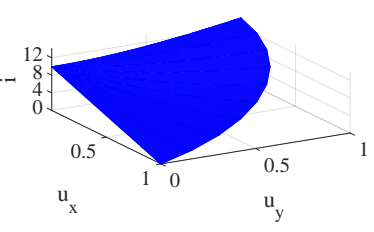

(a) Output current magnitude

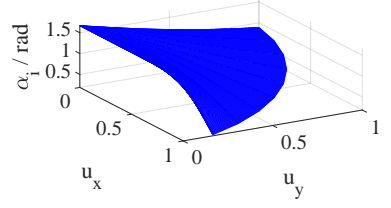

(b) Output current angle
Figure 4: $U-I$ characteristic of an AC grid Thévenin equivalent

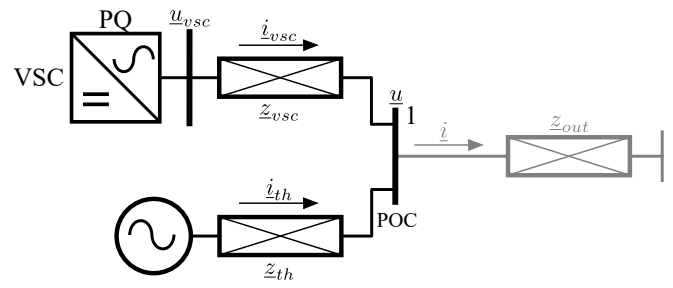

Figure 5: Scheme of a VSC in parallel connection with the AC grid Thévenin Equivalent

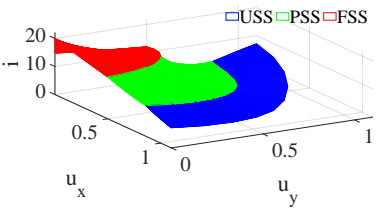

(a) Output current magnitude

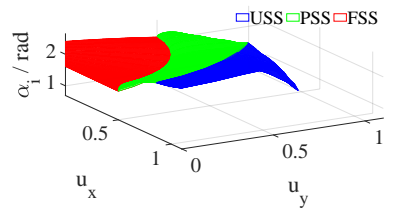

(b) Output current angle
Figure 6: $U-I$ characteristic of a VSC in $P Q$ control connected in parallel with AC grid Thévenin equivalent. Unsaturated (USS), partially saturated (PSS) and fully saturated (FSS) states show the different current saturation states of the converter.

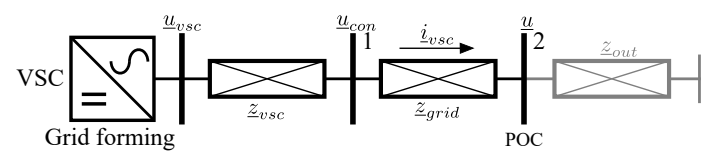

Figure 7: Scheme of a grid-forming VSC

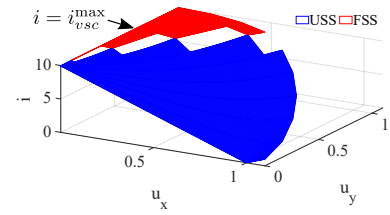

(a) Output current magnitude

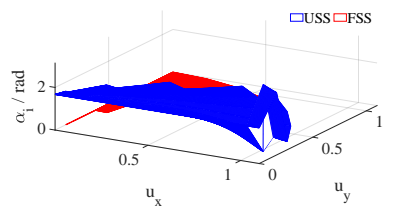

(b) Output current angle
Figure 8: U-I characteristic of grid-forming VSC. Saturated and unsaturated states show the different current saturation states of the converter

of maintaining the voltage stable when the system changes. This can be also analyzed for power systems with important penetration of power electronics, but instead of the very synthetic information needed for the Thévenin equivalent. In this case, a complete map of equivalent impedance is needed 
to quantify the equivalent impedance for the complete system operation range in terms of equilibrium voltages. This is discussed in Section 3.

\section{Impedance mapping and grid strength in power systems with power electronics}

\subsection{General considerations}

In power systems with significant penetration of power electronics, voltages and currents at the selected node can be related in a general form, which connects the voltage and current, as illustrated in Fig. 6:

$$
\underline{i}=F(\underline{u})
$$

where $F$ is a complex function $F: \mathbb{C} \rightarrow \mathbb{C}$. For these systems, an equivalent model (based on the Thévenin equivalent) can be calculated for each equilibrium point, $(\underline{u}, \underline{i}) \in$ $(\mathbb{C}, \mathbb{C})$, as:

$$
\begin{aligned}
& \underline{z}_{e q}(\underline{u})=-\frac{\Delta \underline{u}}{\Delta \underline{i}}=\frac{\Delta \underline{u}}{\Delta F(\underline{u})-F(\underline{u}+\Delta \underline{u})} \\
& \underline{u}_{e q}(\underline{u})=\underline{u}+\frac{\Delta \underline{u}}{\Delta F(\underline{u})-F(\underline{u})} F(\underline{u}+\Delta \underline{u})
\end{aligned}
$$

where the impedance $\underline{z}_{e q}(\underline{u}) \in \mathbb{C}$ defines the relationship between voltage and current variations and therefore defines the strength of the grid. It can be noted that the equivalent impedances will not (in general) be constant for different points. Increments can be made as small as desired and therefore the equivalent impedance can be defined as

$$
\underline{z}_{e q}(\underline{u})=\frac{1}{\underline{y}_{e q}(\underline{u})}=-\frac{1}{d F(\underline{u}) / d \underline{u}}
$$

where the admittance $\underline{y}_{e q} \in \mathbb{C}$ is the complex derivative of the current respect to the voltage. The function $F(\underline{u})$ is complex differentiable (analytic) for a given voltage $\underline{u}$ if the result of the derivative is the same for all the possible angles of $d \underline{u} \in \mathbb{C}$. This can be studied analyzing the derivative for all the angles $\theta$ of $d u$ or alternatively using the CauchyRiemann criterion. The criterion establishes that the complex derivative $d \underline{i} / d \underline{u}$ exists only if the following equalities hold [26]:

$$
\frac{\partial i_{x}}{\partial u_{x}}=\frac{\partial i_{y}}{\partial u_{y}} \text { and }-\frac{\partial i_{x}}{\partial u_{y}}=\frac{\partial i_{y}}{\partial u_{x}}
$$

It will be shown in this Section that this condition does not always hold for the considered system and therefore the derivative depends on the direction of $d \underline{u}$. This fact has important implications in engineering applications as it shows that the system response will be significantly different depending on how (in what direction) the systems currents or voltages are varying. An example of a simple system

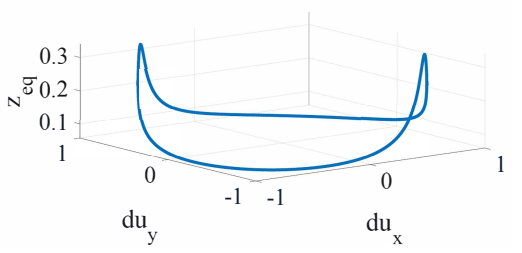

Figure 9: Identified equivalent impedance magnitude with different derivative directions for an example system with a VSC connected in parallel to a Thévenin equivalent with $\underline{u}=$ 1

with a VSC connected in parallel to a Thévenin equivalent is shown in Fig. 9, where the derivatives are shown for different angles.

It is very important to note that $\underline{z}_{e q}(\underline{u})$ is dependant on the voltage $\underline{u}=u_{\angle \alpha_{u}}$ and the direction $\theta$ of the derivative. For each voltage $\underline{u}$, the following impedances can be defined:

- The maximum impedance $\underline{z}_{e q}^{\max }(\underline{u})$ with $d \underline{u}=$ $d u \angle \theta_{z-e q}^{\max }$

- The minimum impedance $\underline{z}_{e q}^{\min }(\underline{u})$ with $d \underline{u}=$ $d u \angle \theta_{z-e q}^{\min }$

- The average impedance $\underline{z}_{e q}^{a v e}(\underline{u})$ with $d \underline{u}=d u \angle \theta_{z-e q}^{a v e}$

Therefore, the equivalent maximum, minimum and average strength of the network depends on the equilibrium point. For a given system, it can be investigated the range of variation of $\underline{z}_{e q}(\underline{u})$ and quantify how much the strength of the network is expected to vary for given voltage (or current) ranges. The overall equivalent maximum impedance $\underline{z}_{e q-o v}^{\max }$, average impedance $\underline{z}_{e q-o v}^{a v e}$ and minimum impedance $\underline{z}_{e q-o v}^{\min }$ can be defined. While these overall impedances will synthesize important information on the system impedance, they will not be sufficient to understand the system steadystate behaviour, as this will require a complete impedance map.

Regarding the concept of short-circuit power, the concept in a general case is not as straight-forward as in the case of the Thévenin equivalent. The short-circuit current can be calculated evaluating $\underline{i}_{s c}=F(0)$ as

$$
\underline{s}_{s c c}=\underline{u}_{e q} \underline{i}_{s c}^{*}=\underline{z}_{e q} F(0) F(0)^{*}=\underline{z}_{e q}|F(0)|^{2}
$$

In the case of systems not defined by a Thévenin equivalent, the concept of short-circuit power does not have the direct meaning of system strength. Short-circuit power gives only information on a specific point of the map, where $\underline{u}=$ 0 . An important consequence of this is that the concept of shortcircuit power cannot be associated to strength of the network, as it common to do in systems which can be modeled with the Thévenin equivalent. 


\subsection{Analysis of system including a Thévenin equivalent and a parallel connected VSC in grid-following control mode}

In the case of a grid including grid-following converters, these converters will be operating either in PQ or PV mode. The converters will be synchronized with a grid formed by a voltage source (typically a Thévenin equivalent or a gridforming converter) and will control the exchange of active and reactive power (associated to voltage control for PV nodes). When converters saturate, they will not be able to keep exchanging the scheduled power and they will remain at maximum current prioritizing $\mathrm{P}$ or $\mathrm{Q}$ exchange depending on the grid codes requirements. The overall equivalent of a system including a Thévenin equivalent plus grid-following converters will diverge from the Thévenin equivalent, being increasingly different when the relative size of the converter increases.

Fig. 5 shows the scheme of an example system containing a VSC in PQ control mode connected in parallel with an AC grid Thévenin equivalent. The studied system is connected with the external system at the point of connection (POC). In this case, the external system is characterized as an impedance, $\underline{z}_{\text {out }}$. The following equations are obtained for the studied system equivalent model:

$$
\begin{aligned}
\underline{u} & =\underline{u}_{t h}-\underline{z}_{t h} \underline{i}_{t h} \\
\underline{u i}_{v s c}^{*} & =p+j q \\
\underline{i} & =\underline{i}_{t h}+\underline{i}_{v s c} \\
\underline{u}_{v s c} & =\underline{u}+\underline{i}_{v s c} \underline{z}_{v s c}
\end{aligned}
$$

where $p$ and $q$ are active and reactive power injection from the VSC.

The grid-following converter can work in the three following states (assuming prioritization of reactive power over active power):

- Normal state, unsaturated state (USS): In this state the converter controls active and reactive power (PQ mode) or active power and $\mathrm{AC}$ voltage (PV mode). The voltage $\underline{u}$ and $\underline{i}_{v s c}$ is maintained within limits.

- Partial saturated state (PSS): The converter works at maximum current $i_{\max }$, supplying all the required reactive power and a fraction of the required active power.

- Fully saturated state (FSS): The converter has arrived to the maximum current and it maintains maximum current $i_{\max }$ with $p=0$ and supplying a fraction of the required reactive power.

Additional equations are included to characterize the VSC operation gathering the control modes and various potential current saturation states. In this case, the VSC in PQ control can be divided into the following current saturation states:

$$
\begin{cases}p=p_{r e f} ; q=q_{r e f} & \text { if unsaturated (USS) } \\ q=q_{r e f} ; i_{v s c}=i_{v s c}^{\max } & \text { if partially saturated (PSS) } \\ p=0 ; i_{v s c}=i_{v s c}^{\max } & \text { if fully saturated (FSS) }\end{cases}
$$

where $p_{\text {ref }}$ and $q_{\text {ref }}$ are active and reactive power reference of the VSC, $i_{v s c}^{\max }$ is the VSC nominal current.

The identified equivalent impedance of the studied system can be expressed as follows:

$$
\frac{1}{\underline{z}_{e q}}=-\frac{d \underline{i}}{d \underline{u}}=-\frac{d \underline{i}_{v s c}+d \underline{i}_{t h}}{d \underline{u}}=-\frac{d \underline{i}_{v s c}}{d \underline{u}}+\frac{1}{\underline{z}_{t h}}
$$

\subsubsection{Impedance $\underline{z}_{e q}$ in unsaturated state (USS)}

In USS, from (12) we can obtain $\underline{i}_{v s c}=\left(p_{\text {ref }}-j q_{r e f}\right) / \underline{u}^{*}$ and

$$
\frac{d \underline{i}_{v s c}}{d \underline{u}}=\left(p_{r e f}-j q_{r e f}\right) \frac{d}{d \underline{u}} \frac{1}{\underline{u}^{*}}=\left(p_{r e f}-j q_{r e f}\right) \frac{-1}{\underline{u}^{2}} \frac{d \underline{u}^{*}}{d \underline{u}}
$$

where the derivative $d \underline{u}^{*} / d \underline{u}$ is not analytic as it has a different result depending on the direction of $d \underline{u}=d u_{\angle \theta}$.

$$
\frac{d \underline{u}^{*}}{d \underline{u}}=\lim _{u \rightarrow 0} \frac{u \cos \theta-j u \sin \theta}{u \cos \theta+j u \sin \theta}=\left(e^{j 2 \theta}\right)^{*}
$$

where $d \underline{u}^{*} / d \underline{u}=\left(e^{j 2 \theta}\right)^{*}$ maintains a modulus of 1 but the angle depends on the angle $\theta$ of $d \underline{u}$.

The non-differentiability can be verified with the Cauchy-Riemann equations criterion, which is not satisfied. The fact that the complex derivative does not exist (the function is not analytic) has some important consequences and it leads to the existence of different derivatives for each voltage depending on the direction of differentiation. The converter impedance can be expressed as

$$
\underline{z}_{u s s}(\underline{u}, \theta)=\frac{\underline{u}^{2}}{\left(p_{r e f}-j q_{r e f}\right)\left(e^{j 2 \theta}\right)^{*}}
$$

The equivalent overall impedance can be generally written as

$\frac{1}{\underline{z}_{e q}}=-\frac{d \underline{i}}{d \underline{u}}=\frac{1}{\underline{z}_{u s s}(\underline{u}, \theta)}+\frac{1}{\underline{z}_{t h}}=\frac{p_{r e f}-j q_{r e f}}{\underline{u}^{2}}\left(e^{j 2 \theta}\right)^{*}+\frac{1}{\underline{z}_{t h}}$

which yields two important results:

- Equivalent impedance changes for different voltages $\underline{u}$. 
- The equivalent impedance for each voltage $\underline{u}$ depends on the direction of the complex derivative. Therefore, for each voltage $\underline{u}$ there are multiple different values of the derivative for each angle $\theta$.

Fig. 10 shows example results on how the impedance changes for different derivative angles and voltages (different voltage modules are considered with $\alpha_{u}=0 E$ ). The results are obtained evaluating numerically the derivative in the V-I characteristic. The numerical results match with (17).

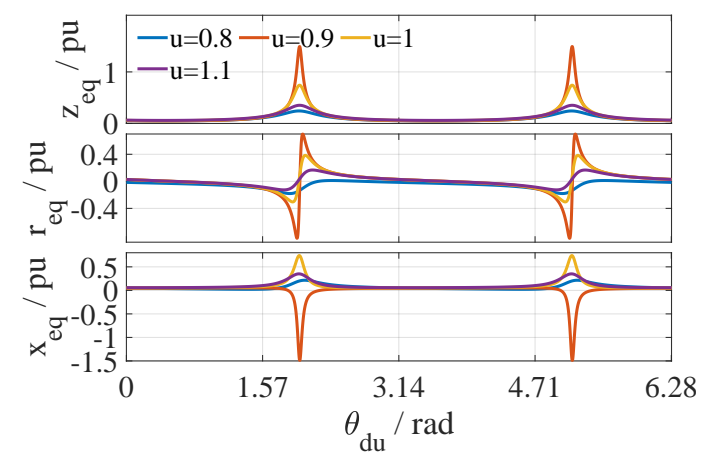

Figure 10: Identified equivalent impedance with different derivative directions for VSC operated in USS

\subsubsection{Impedance $\underline{z}_{e q}$ in fully saturated state (FSS)}

For FSS, active power is set to zero $(p=0)$ and the converter operates at maximum current to inject the maximum reactive power. Therefore, $\underline{i}_{v s c}$ is shifted $90^{\circ}$ from $\underline{u}=u_{\angle \alpha_{u}}$ :

$$
\begin{aligned}
& \underline{i}_{v s c}(\underline{u})=i_{v s c}^{\max } \angle \alpha_{i}=i_{v s c \angle \alpha_{u}+\pi / 2}^{\max } \\
& \underline{i}_{v s c}(\underline{u})=-i_{v s c}^{\max } \sin \left(\alpha_{u}\right)+j i_{v s c}^{\max } \cos \left(\alpha_{u}\right) \\
& \frac{d \underline{i}-v s c}{d \underline{u}}=\lim _{d \underline{u} \rightarrow 0} \frac{i_{v s s c}^{\max }}{d u}\left(-\sin \left(\alpha_{u+d u}\right)+j \cos \left(\alpha_{u+d u}\right)-\right. \\
& \left.-\left(-\sin \left(\alpha_{u}\right)+j \cos \left(\alpha_{u}\right)\right)\right)
\end{aligned}
$$

The following mathematical relationships are used to calculate $d \underline{i}_{v s c} / d \underline{u}$ :

$$
\begin{aligned}
& \sin \left(\alpha_{u}\right)=\frac{u_{y}}{\sqrt{u_{x}^{2}+u_{y}^{2}}}, \cos \left(\alpha_{u}\right)=\frac{u_{x}}{\sqrt{u_{x}^{2}+u_{y}^{2}}} \\
& \sin \left(\alpha_{u+d u}\right)=\frac{u_{y}+d u_{y}}{\sqrt{\left(u_{y}+d u_{y}\right)^{2}+\left(u_{x}+d u_{x}\right)^{2}}} \\
& \cos \left(\alpha_{u+d u}\right)=\frac{u_{x}+d u_{x}}{\sqrt{\left(u_{y}+d u_{y}\right)^{2}+\left(u_{x}+d u_{x}\right)^{2}}}
\end{aligned}
$$

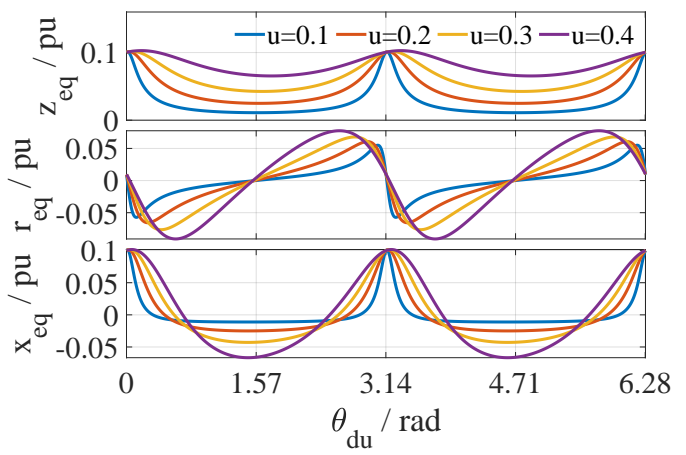

Figure 11: Identified equivalent impedance with different derivative directions for VSC operated in FSS

Replacing (21)-(23) in (20), using $d u_{x}=d u \cos \theta$ and $d u_{y}=d u \sin \theta$ (where $\theta$ is the angle of the derivative), the complex derivative $d \underline{i}_{-v s c} / d \underline{u}$ can be expressed as

$$
\begin{array}{r}
\frac{d \underline{i}_{-v s c}^{v s c}}{d \underline{u}}=\lim _{d u \rightarrow 0} \frac{i_{\max }}{d u(\cos \theta+j \sin \theta)} \\
{\left[\frac{-\left(u_{y}+d u \sin \theta\right)+j\left(u_{x}+d u \cos \theta\right)}{\sqrt{u^{2}+2 u_{x} d u \cos \theta+2 u_{y} d u \sin \theta+d u^{2}}}+\frac{u_{y}-j u_{x}}{u}\right]}
\end{array}
$$

where part of the expression is a function that can be approximated with the following Taylor first order polynomial as

$$
\frac{A+B x}{\sqrt{C+D x+E x^{2}}} \approx \frac{A}{\sqrt{C}}+\frac{B C-A D / 2}{C \sqrt{C}} x+\ldots
$$

Using this approximation in equation (24):

$$
\frac{d i_{-v s c}}{d \underline{u}} \approx \frac{i_{v s c}^{\max }}{u}\left(e^{j \pi / 2}-e^{j\left(\alpha_{u}-\theta+\pi / 2\right)} \cos \left(\alpha_{u}-\theta\right)\right)
$$

where it is clear that the complex derivative $d \underline{i}_{-v s c} / d \underline{u}$ does not exist (it is not analytic), but the the derivatives in different directions for different voltages $u$ can be calculated using the previous expression. It can be seen that depending on the direction of $\theta$ completely different results are obtained.

The FSS impedance $\left.\underline{z}_{f s s} \underline{u}, \theta\right)$ can be defined as the grid equivalent impedance associated the converter in FSS state.

$$
\underline{z}_{f s s}(\underline{u}, \theta) \approx \frac{u}{i_{v s c}^{\max }}\left(e^{j \pi / 2}+e^{\alpha_{u}-\theta-\pi / 2} \cos \left(\alpha_{u}-\theta\right)\right)^{-1}
$$

The equivalent impedance can be expressed as a function of $\underline{u}=u_{\angle \alpha_{u}}$ and $\theta$ as:

$$
\frac{1}{\underline{z}_{e q}}=\frac{1}{\underline{z}_{t h}}+\frac{1}{\underline{z}_{f s s}(\underline{u}, \theta)}
$$

Fig. 11 includes an example that shows how the equivalent impedance changes for different derivation angles and voltages (different voltage modules are considered with $\left.\alpha_{u}=0 E\right)$. The results are obtained evaluating numerically the derivative in the V-I characteristic. The numerical results match with (27) and (28). 


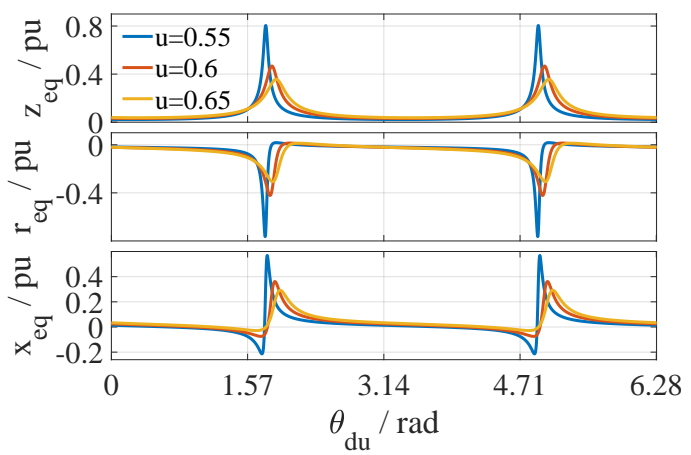

Figure 12: Identified equivalent impedance with different derivative directions for VSC operated in PSS

\subsubsection{Impedance $\underline{z}_{e q}$ in partially saturated state (PSS)} For PSS,

$$
\stackrel{i}{u s c}=i_{\max \angle \alpha_{i}}=i_{\max \angle \alpha_{u}+\delta_{u}}
$$

where $\delta_{=} \alpha_{u}-\alpha_{i}$ is the angle between voltage and current.

The VSC current derivation of voltage can be expressed as:

$$
\frac{d \underline{i} v s c}{d \underline{u}}=\lim _{d \underline{u} \rightarrow 0} \frac{i_{\max \angle \alpha_{u+d u}+\delta_{u+d u}}-i_{\max \angle \alpha_{u}+\delta_{u}}}{d \underline{u}}
$$

which can be simplified similar a procedure similar to the FSS case. Applying similar simplifications and linealization around $d u=0$, it leads to:

$$
\underline{z}_{p s s}(\underline{u}, \theta) \approx \frac{p e^{j \theta}+q e^{j(\theta-\pi / 2)}+\kappa e^{j \alpha_{u}} \frac{u^{2} i_{\max }^{2}}{p}-2 \kappa \underline{\sigma}}{e^{j \theta} u^{2}}
$$

with $\kappa=\cos \alpha_{u} \cos \theta+\sin \alpha_{u} \sin \theta, p^{2}=u^{2} i_{\text {max }}^{2}-q^{2}$, and $\underline{\sigma}=p e^{j \alpha_{u}}+q e^{j\left(\alpha_{u}-\pi / 2\right)}$. The derivative is clearly not analytic and depends on both the voltage $\underline{u}$ and the angle of derivation $\theta$.

The equivalent impedance can be expressed as a function of $\underline{u}=u_{\angle \alpha_{u}}$ and $\theta$ as:

$$
\frac{1}{\underline{z}_{e q}}=\frac{1}{\underline{z}_{t h}}+\frac{1}{\underline{z}_{p s s}(\underline{u}, \theta)}
$$

Fig. 12 includes an example that shows how the equivalent impedance changes for different derivation angles and voltages (different voltage modules are considered with $\alpha_{u}=0 \mathcal{E}$ ). The results are obtained evaluating numerically the derivative in the V-I characteristic. The numerical results match with (31) and (32).

\subsection{Grid-forming converter}

In the case of a grid-forming converter, the system behavior is clearly different in the two following states:

- Normal state, unsaturated (USS): The voltage $\underline{u}$ is maintained at the converter terminals for any current. Therefore, the converter behaves as an ideal voltage source, with $\underline{z}_{e q}=0$. If there is some impedance in the line, this line impedance will be the equivalent impedance $\underline{z}_{e q}$.
- Fully saturated state (FSS): The converter has arrived to the maximum current and it maintains maximum current $i_{\max }$ regardless the voltage. In this case, the behaviour corresponds to an ideal current source, with $\underline{y}_{e q}=0$ and $\underline{z}_{e q}=\infty$.

The scheme of a grid-forming VSC is shown in Fig. 7. The voltage at bus $1, \underline{u}_{c o n}$, is regulated at the reference value by the VSC if it is operating in normal state. The studied system, which is composed of the grid-forming VSC and a series impedance, $\underline{z}_{g r i d}$, is connected to the external system at the POC. The following equations are obtained for characterize the studied system:

$$
\begin{aligned}
\underline{u}_{c o n}-\underline{u} & =\underline{z}_{g r i d} \underline{i}_{v s c} \\
\underline{u}_{v s c}-\underline{u}_{c o n} & =\underline{z}_{v s c-v s c}
\end{aligned}
$$

Additional equations will be included modeling the operation of the grid-forming VSC in two potential current saturation states:

$$
\begin{cases}\underline{u}_{c o n}=\underline{u}_{r e f} & \text { if USS } \\ \underline{i}_{v s c}=i_{v s c}^{\max } \angle \alpha_{i}^{r e f} & \text { if FSS }\end{cases}
$$

where $\underline{u}_{r e f}$ is the voltage reference of the VSC and $\alpha_{i}^{r e f}$ is the reference of current angle for VSC operation in saturated state.

The equivalent impedance of the studied system can be identified as:

$$
\underline{z}_{e q}=-d \underline{u} / d \underline{i}_{v s c}
$$

When the VSC is operating in normal state and regulating a constant voltage, $\underline{u}_{c o n}=\underline{u}_{r e f}$, at bus 1, CauchyRiemann equations hold for (36) with the partially derivative elements obtained as follows:

$$
\left\{\begin{array}{l}
\partial u_{x} / \partial i_{v s c x}=\partial u_{y} / \partial i_{v s c y}=-z_{\text {grid } x} \\
\partial u_{x} / \partial i_{v s c y}=-\partial u_{y} / \partial i_{v s c x}=z_{g r i d}
\end{array}\right.
$$

As a result, the identified equivalent impedance of the studied system is the same for different directions of voltage derivatives at the POC and can be obtained as:

$$
\underline{z}_{e q}=-\partial u_{x} / \partial i_{v s c x}-j \partial u_{x} / \partial i_{v s c y}=\underline{z}_{g r i d}
$$

When the VSC is operating in saturated state, the current remains constant for any voltage deviation. Therefore $\frac{d \underline{i}_{v s c}}{d \underline{u}}=0$ and $\underline{z}_{e q}=\infty$.

\section{Methodology to obtain the impedance map}

Algorithm 1 describes the procedure to obtain the equivalent impedance map. The studied power system is defined with the function $F$ which maps voltage to current. Voltage at the POC is tested for a set of voltages $\underline{u}$ with $N_{u-\bmod }$ different magnitudes and $N_{u-a n g l e}$ different phase angles. For 
each voltage, $\underline{u}$, the voltage derivatives are tested for $N_{\theta}$ directions with a differential of voltage $d u=\varepsilon$ which is set to $10^{-6} \mathrm{pu}$. For each voltage $\underline{u}, N_{\theta}$ values of impedance $\underline{z}_{e q}(\underline{u}, \theta)$ are obtained, which can be equal or different depending on the case. The obtained impedances are used to calculate the maximum impedance $\underline{z}_{e q}^{\max }(\underline{u})$, the minimum impedance $\underline{z}_{e q}^{\min }(\underline{u})$ and the average impedance $\underline{z}_{e q}^{a v e}(\underline{u})$ for each voltage. Once all the impedances are calculated for all the voltages, the overall equivalent maximum impedance $\underline{z}_{e q-o v}^{\max }$, overall average impedance $\underline{z}_{e q-o v}^{a v e}$ and overall minimum impedance $\underline{z}_{e q-o v}^{\mathrm{min}}$ are calculated.

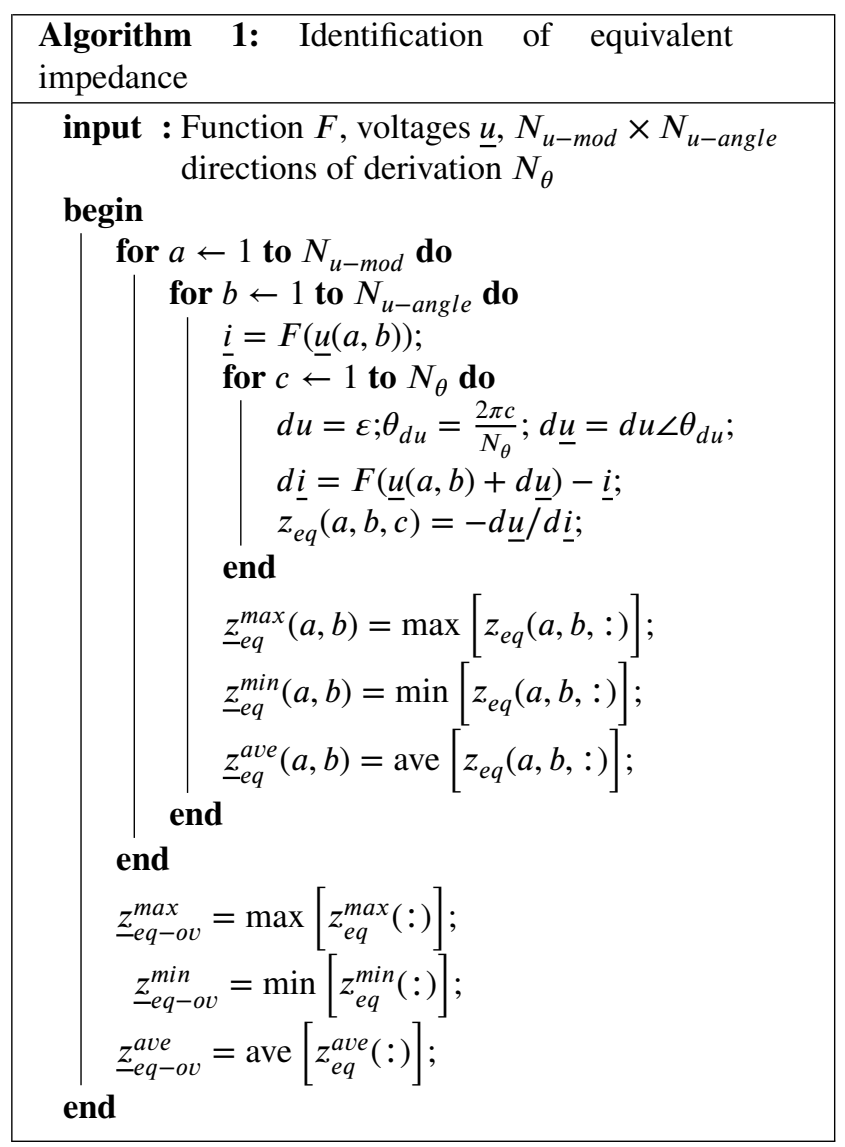

\section{Numerical Case Studies}

The present Section presents numerical analyses on the different configurations discussed in the previous Sections. The systems analyzed include an ideal Thévenin equivalent, different cases of a grid-following VSC in parallel with a Thévenin equivalent and a grid-forming converter.

\subsection{Thévenin equivalent (Case 1)}

The Thévenin equivalent (shown in Fig. 3 and Fig. 4) has been analyzed in Case 1. As it is shown in equation (1), the obtained Thévenin impedances matches exactly the parameters of the Thévenin equivalent for all the voltages and for all the derivation angles. The results are shown in Figs 13 . The identified equivalent impedance $\underline{z}_{e q}$ is equal to the Thévenin impedance $\underline{z}_{t h}$ for all different equilibrium voltages and different directions of voltage derivatives.
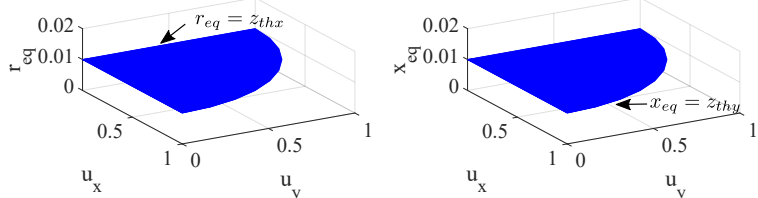

Figure 13: Identified equivalent resistance and reactance of AC grid Thévenin equivalent

\subsection{Small grid-following VSC in parallel with a Thévenin equivalent (Case 2)}

In case 2, A small grid-following VSC in parallel with a Thévenin equivalent (shown in Fig. 5) is analyzed considering the parameters of the Appendix. It can be noted that the obtained impedances change for angles of derivation $\theta$ for each voltage $\underline{u}=u_{x}+j u_{y}$. The maximum $z_{e q}^{\max }$, minimum $z_{e q}^{\min }$ and average $z_{e q}^{a v e}$ impedances for each voltage are shown in Fig. 14. The Fig. shows a peak in FSS.

Rearranging (28), the equivalent impedance $\underline{z}_{e q}$ can be expressed as:

$$
\underline{z}_{e q}=\underline{z}_{t h}\left(1+\frac{\underline{z}_{t h}}{\underline{z}_{f s s}(\underline{u}, \theta)}\right)^{-1}
$$

where it can be noted that $\underline{z}_{e q}=\infty$ when $\underline{z}_{t h}=-\underline{z}_{f s s}(\underline{u}, \theta)$. The voltage $\underline{u}$ which will provoke this peak can be calculated as

$$
\begin{aligned}
& u=i_{v s c}^{\max } z_{t h} \quad \forall \alpha_{u} \\
& \theta=\frac{-\log \left( \pm e^{\alpha_{u}} \sqrt{\left.-2 j e^{\alpha_{z_{t h}}+j e^{2 \alpha_{z_{t h}}}}\right)}\right.}{j e^{\alpha_{z_{t h}}+2}}
\end{aligned}
$$

where $\alpha_{z_{t h}}$ is the impedance angle of $\underline{z}_{t h}$.

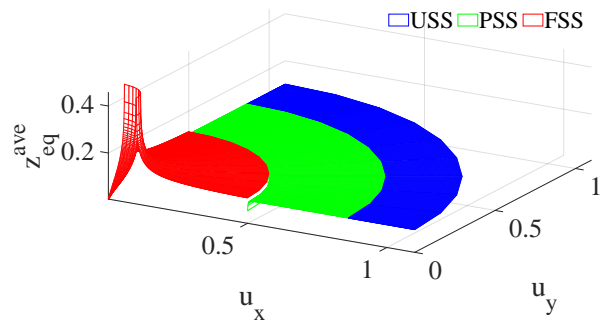

(a) $z_{e q}^{a v e}$

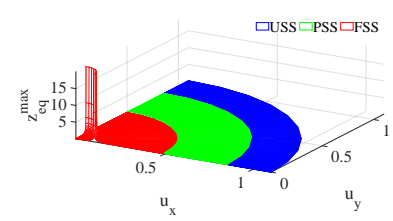

(b) $z_{e q}^{\max }$

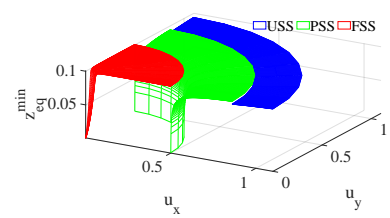

(c) $z_{e q}^{\min }$
Figure 14: Identified equivalent impedance map of Case 2 


\subsection{Large grid-following VSC in parallel with a Thévenin equivalent (Case 3)}

In case 3, A large grid-following VSC in parallel with a Thévenin equivalent (shown in Fig. 5 and 6) is analyzed considering the parameters of the Appendix. It can be noted that the obtained impedances change for angles of derivation $\theta$ for each voltage $\underline{u}=u_{x}+j u_{y}$. The maximum $z_{e q}^{\max }$, minimum $z_{e q}^{\min }$ and average $z_{e q}^{a v e}$ impedances for each voltage are shown in Fig. 15. A peak in the impedance for USS state can be noticed. Using (16) and (17), the equilibrium voltage magnitude and direction angle of voltage derivative corresponding to the infinite equivalent impedance can be obtained as:

$$
\begin{aligned}
& u=\left|p_{\text {ref }}+j q_{\text {ref } f}\right| / z_{t h} \quad \forall \alpha_{u} \\
& \theta=-\alpha_{z_{t h}} / 2
\end{aligned}
$$

where $\alpha_{z_{t h}}$ is the impedance angle of $\underline{z}_{t h}$. The peak obtained in FSS for small VSC is not obtained in a large VSC because the voltage fulfilling the conditions is outside the FSS range.

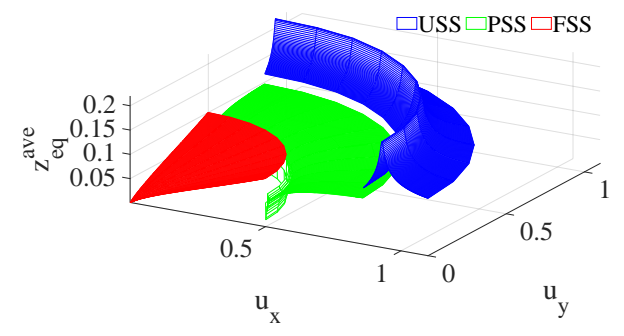

(a) $z_{e q}^{a v e}$

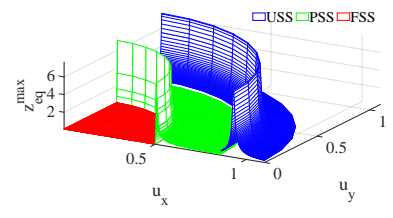

(b) $z_{e q}^{\max }$

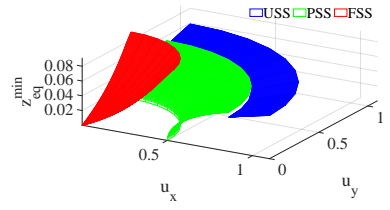

(c) $z_{e q}^{\min }$
Figure 15: Identified equivalent impedance map of Case 3

\subsection{Sweep different penetrations of VSC in parallel with a Thévenin equivalent}

In order to understand the behaviour of a VSC in parallel with a Thévenin equivalent for different sizes of the VSC relative to the short-circuit power of the Thévenin equivalent, a sweep for different ratios $\eta=s_{v s c} / s_{s c}$ have been conducted. Fig. 16 shows that for the different ratios, the equivalent average impedance tends to 0 for low voltages and tends to $z_{t h}$ for high voltages (the dashed blue line with ratio 0 is a pure Thévenin equivalent). Ratio has been tested from 0 to 2 while $u$ is tested from 0 to 2 pu for each ratio. It can be noticed that for all different ratios there is a peak in the impedance (as analyzed in the previous specific cases) which corresponds to different voltages depending on the ratio. This maximum is obtained for different saturation states depending on the ratio. Fig. 16 shows how the average, maximum and minimum impedance change for different ratios. The Fig. also shows the average overall impedance change for different ratios.
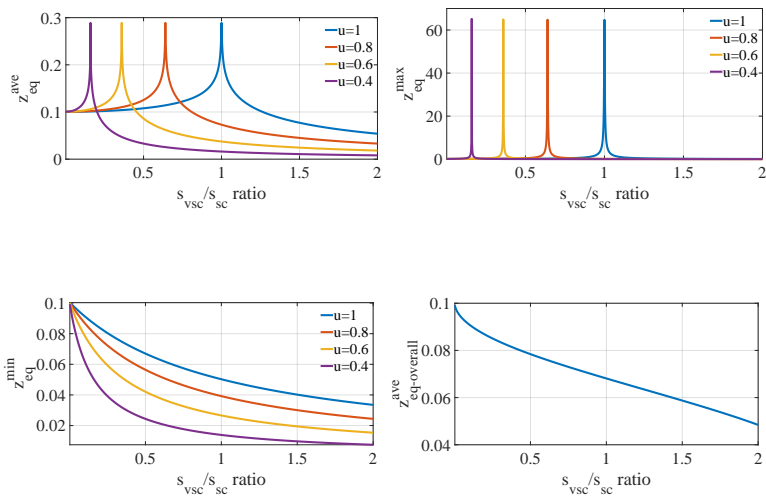

Figure 16: Identified equivalent impedances with different $p_{v s c} / s_{s c}$

\subsection{Grid-forming converter (Case 4)}

A grid-forming converter (shown in Fig. 7 and Fig. 8) is analyzed. As it has been discussed in Section 3, the results shown in Fig. 17 indicate clearly than the equivalent impedance corresponds to the line impedance connected in series with the grid-forming converter until it arrives to saturation. In this case the impedance becomes infinity as the converter behave as a pure current source.

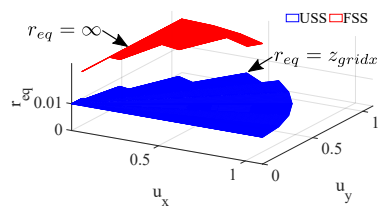

(a) $r_{e q}$

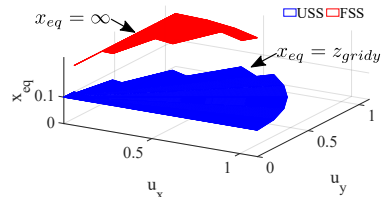

(b) $x_{e q}$
Figure 17: Equivalent resistance and reactance for a gridforming converter

\subsection{Modified IEEE 9-bus system with two VSCs (Case 5)}

This case study deals with a test system constructed based on IEEE 9-bus system as shown in Fig. 18. Two grid-following VSCs are connected at buses 2 and 3, both operating in PQ control and following the set points shown in the Appendix. Three load impedances are connected at buses 5, 6 and 8. The equivalent of this test system is characterized from bus 9 (which is defined as the POC in the studied system). The voltage-current characteristic of the test system is shown in Fig. 19 where six combinations of converters' current-saturation states are presented. The identified equivalent impedance corresponding to different output voltage magnitudes and angles are shown in Fig. 20. It can be observed that both the average $z_{e q}^{a v e}$ and maximum 


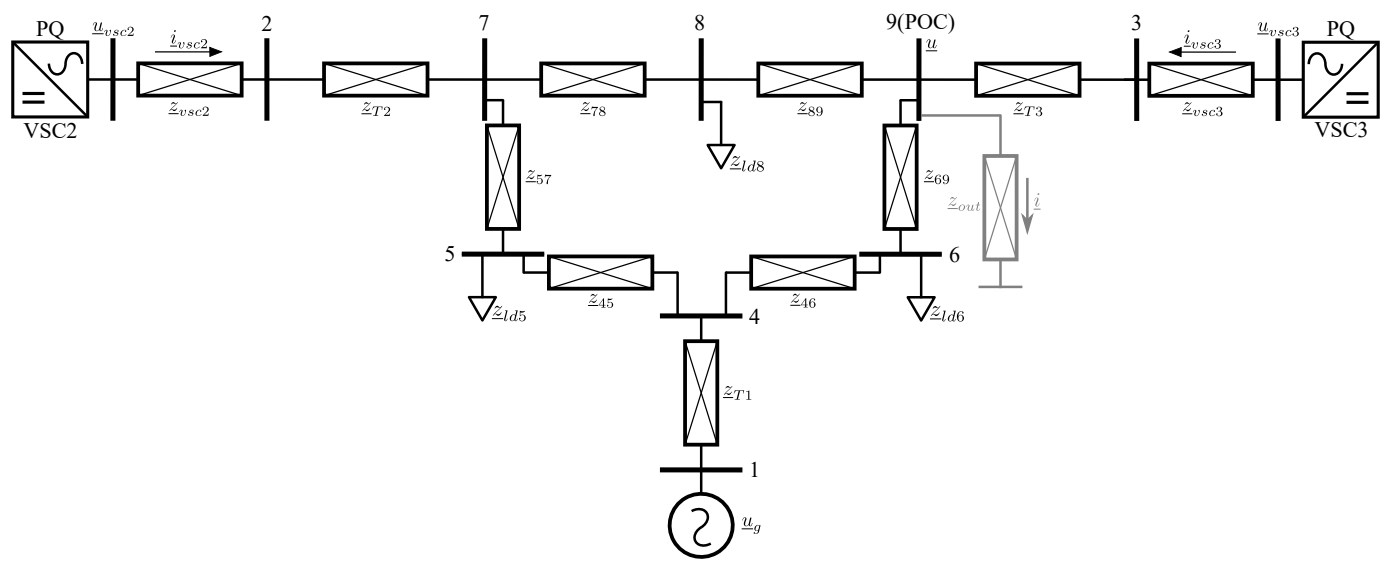

Figure 18: Scheme of the Test System Studied in Case 5

$z_{\text {eq }}^{a v e}$ impedances achieve peak value with a low voltage level at bus 9 where both VSCs are operating in FSS.

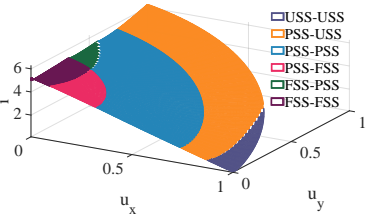

(a) Output current magnitude

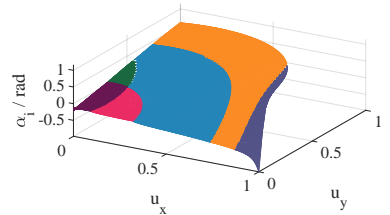

(b) Output current angle
Figure 19: $U-I$ characteristic of Case 5

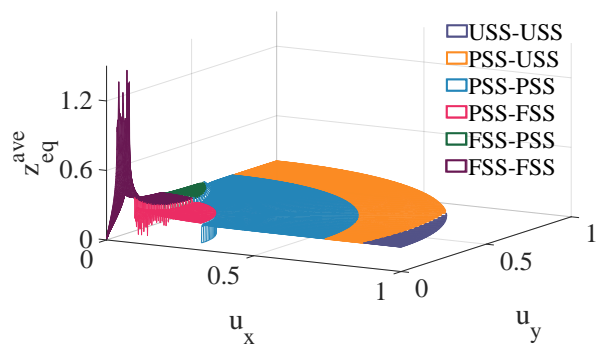

(a) $z_{e q}^{a v e}$

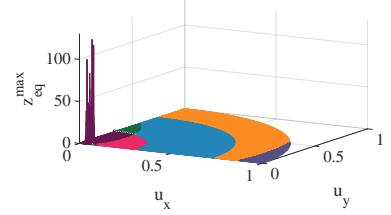

(b) $z_{e q}^{\max }$

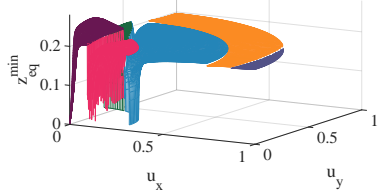

(c) $z_{e q}^{\min }$
Figure 20: Identified equivalent impedance map of Case 5

\section{Conclusions}

The paper has analyzed the concept of grid strength for systems with significant penetration of power electronics, focusing on the computation of equivalent grid impedance. The work has shown that using a Thévenin equivalent is not sufficient to take into account the different saturation states of power converters. The consequence of this is that classical definitions of grid strength based on short-circuit power at the point of connection fail to describe the system behavior. The paper has proposed an alternative, based on mapping the impedance for all the different possible voltages at the point of connection and analyzing how the impedance depend on the voltage and angle of derivation for different systems. These systems include a grid-following converter in parallel with a Thévenin equivalent and a gridforming converter. In all the cases, the paper considers the different saturation states of the converter. Numerical results and example case studies have been included. For grid-following converters in parallel with a Thévenin equivalent, it has been shown that the impedance is highly depending on the voltage and the derivation angle. The overall impedance tends to decrease as the power of the converter increases. In grid-forming converters, there are two clear states: constant impedance for normal unsaturated state and infinite impedance when the converter is saturated and injects constant maximum current.

\section{A. Test System Parameters}

All the numerical calculations have been implemented in per-unit with a Thévenin equivalent of parameters $\underline{u}_{t h}=1$ and $\underline{z}_{t h}=0.01+\mathrm{j} 0.1$. In Case 2 the converter operates with $p_{\text {ref }}=0.7, q_{\text {ref }}=0.5$ and $i_{\max }^{v s c}=1$. In Case 3 the converter operates with $p_{r e f}=7, q_{r e f}=5$ and $i_{\max }^{v s c}=10$. In Case 4 the converter operates with $u_{r e f}=1$ and $i_{\max }^{u s c}=1$. In case 5, VSC2 operates with $p_{\text {ref } 2}=0.5, q_{\text {ref } 2}=0.3$ and $i_{\max }^{v s c 2}=0.6$ while VSC3 operates with $p_{\text {ref } 3}=0.2, q_{\text {ref } 3}=0.1$ and $i_{\max }^{v s c 3}=0.3$.

\section{References}

[1] J.C. Das. Power System Analysis - Short-Circuit Load Flow and Harmonics. 2010.

[2] Jürgen Schlabbach. Short-circuit currents. IET, 2005.

[3] CIGRE Working Group C4.503. Power system technical perfor- 
mance - Power system test cases for EMT-type simulation studies. Technical Report December, CIGRE, 2019.

[4] Hani Saad, Albane Schwob, and Yannick Vernay. Study of resonance issues between HVDC link and power system components using EMT simulations. In 20th Power Systems Computation Conference, PSCC 2018. Institute of Electrical and Electronics Engineers Inc., aug 2018.

[5] H Saad, Y Vernay, S Dennetiere, P Rault, and B Clerc. System Dynamic Studies of Power Electronics Devices with Real-Time Simulation - A TSO operational experience. In CIGRE session 2018, Paris, 2018.

[6] North American Electric Reliability Corporation. Integrating Inverter-Based REsources into Low Short Circuit Strength Systems, 2017.

[7] National Grid. Grid Code Review Panel Report from the Grid Code Reactive Review Sub-group, 2001.

[8] National Energy Administration of China. GB 38755-2019: Guide on Security and Stability for Power System (in Chinese), 2019.

[9] CIGRE Working Group 14.05. On Voltage and Power Stability in AC/DC Systems, 2003.

[10] Zhaosen Chai, Hui Li, Xiangjie Xie, Mohamed Abdeen, Tian Yang, and Kun Wang. Output impedance modeling and grid-connected stability study of virtual synchronous control-based doubly-fed induction generator wind turbines in weak grids. International Journal of Electrical Power and Energy Systems, 126(PA):106601, 2021.

[11] Di Wu, Gangan Li, Milad Javadi, Alexander M. Malyscheff, Mingguo Hong, and John Ning Jiang. Assessing Impact of Renewable Energy Integration on System Strength Using Site-Dependent Short Circuit Ratio. IEEE Transactions on Sustainable Energy, 9(3):10721080, 2018.

[12] Jing Wang, Ilya Tyuryukanov, and Antonello Monti. Design of a novel robust current controller for grid-connected inverter against grid impedance variations. International Journal of Electrical Power and Energy Systems, 110(January):454-466, 2019.

[13] A. Vijayakumari, A. T. Devarajan, and N. Devarajan. Decoupled control of grid connected inverter with dynamic online grid impedance measurements for micro grid applications. International Journal of Electrical Power and Energy Systems, 68:1-14, 2015.

[14] Yang Zhang, Shun Hsien Fred Huang, John Schmall, Jose Conto, Jeffrey Billo, and Ehsan Rehman. Evaluating system strength for large-scale wind plant integration. IEEE Power and Energy Society General Meeting, 2014-October(October), 2014.

[15] Xing Wang Chen and Guan-Lin. Research on limitation of the multiinfeed short circuit ratio. Asia-Pacific Power and Energy Engineering Conference, APPEEC, 2016-December:712-715, 2016.

[16] Ke Chen, Jianyu Luo, Ting Zhou, Kaiming Luo, Sicheng Wang, and Dechun Zang. Summary of Research on Multi-infeed Short Circuit Ratio in AC and DC Systems. 2nd IEEE Conference on Energy Internet and Energy System Integration, EI2 2018 - Proceedings, 2018.

[17] Xiaojun Ni, Aniruddha M. Gole, Chengyong Zhao, and Chunyi Guo. An Improved Measure of AC System Strength for Performance Analysis of Multi-Infeed HVdc Systems Including VSC and LCC Converters. IEEE Transactions on Power Delivery, 33(1):169-178, 2018.

[18] Xing Wang Chen, Guan-Lin, Sheng Lei, and Bao Rong Zhou. Effective short circuit ratio including impact of STATCOM. AsiaPacific Power and Energy Engineering Conference, APPEEC, 2016December:800-804, 2016.

[19] Yan Liu and Zhe Chen. A flexible power control method of VSCHVDC link for the enhancement of effective short-circuit ratio in a hybrid multi-infeed HVDC system. IEEE Transactions on Power Systems, 28(2):1568-1581, 2013

[20] Wei Dong, Huanhai Xin, Di Wu, and Linbin Huang. Small Signal Stability Analysis of Multi-Infeed Power Electronic Systems Based on Grid Strength Assessment. IEEE Transactions on Power Systems, 34(2):1393-1403, 2019.

[21] Feng Zhang, Huanhai Xin, Di Wu, Zhen Wang, and Deqiang Gan. Assessing strength of multi-infeed LCC-HVDC systems using generalized short-circuit ratio. IEEE Transactions on Power Systems,
34(1):467-480, 2019.

[22] Linbin Huang, Huanhai Xin, Zhiyi Li, Ping Ju, Hui Yuan, and Guanzhong Wang. Identification of Generalized Short-Circuit Ratio for On-Line Stability Monitoring of Wind Farms. IEEE Transactions on Power Systems, 35(4):3282-3285, 2020.

[23] IEEE Power Engineering Society. IEEE Guide for Planning DC Links Terminating at AC Locations Having Low Short-Circuit Capacities. Technical report, 1997.

[24] International Electrotechnical Commission (IEC). Short-circuit currents in three-phase a.c. systems - Part 0: Calculation of currents (IEC 60909-0:2016). Technical report, 2016.

[25] CIGRE Working Group 14.07 and IEEE Working Group 15.05.05. Guide for Planning DC Links Terminating at AC System Locations Having Low Short-circuit Capacities. Part I: AC/DC Interaction Phenomena. Technical Report June, 1992.

[26] Walter Rudin. Real and Complex Analysis. McGraw-Hill, 1986. 